\title{
Construção do Estado da Arte em Estratégias Híbridas para Raciocínio de Contexto: uma Abordagem explorando Revisão Sistemática da Literatura
}

\author{
Roger S. Machado ${ }^{1}$, Ricardo B. Almeida ${ }^{1}$, João Ladislau Lopes ${ }^{2}$, \\ Ana Marilza Pernas ${ }^{1}$, Adenauer C. Yamin ${ }^{1}$ \\ ${ }^{1}$ Programa de Pós-Graduação em Computação (PPGC) \\ Universidade Federal de Pelotas (UFPel) \\ ${ }^{2}$ Instituto Federal Sul-rio-grandense (IFSul) \\ \{rdsmachado, rbalmeida, marilza, adenauer\}@inf.ufpel.edu.br \\ joaolopesecavg.ifsul.edu.br
}

\begin{abstract}
Hybrid proposals for context reasoning stand out in the literature, these proposals allow the use of two or more reasoning strategies. Motivated by this scenario, this paper aims to build the state of the art, systematizing research trends in Context Awareness. A Systematic Literature Review is explored, contributing to the identification of relevant works in the area, as well as the specification of criteria for its selection. In this review, we analyze works published between 2012 and 2017. During the review process, 2952 papers were identified, and at the end, seven works that proposed hybrid strategies were selected. The selected works are presented, discussed, and a comparison between them is performed.
\end{abstract}

Resumo. As propostas híbridas para raciocínio de contexto vêm ganhando destaque na literatura, as mesmas permitem a utilização de duas ou mais estratégias de raciocínio. Motivado por este cenário, esse artigo tem o objetivo de construir o estado da arte, sistematizando tendências de pesquisa em Ciência de Contexto. É explorada uma Revisão Sistemática da Literatura, contribuindo tanto pela identificação de trabalhos relevantes na área, bem como, pela especificação de critérios para sua seleção. Nessa revisão são analisados trabalhos publicados entre 2012 e 2017. Durante o processo de revisão foram identificados 2952 trabalhos, sendo selecionados ao final sete trabalhos que propõem estratégias híbridas. Os trabalhos selecionados são apresentados, discutidos, e uma comparação entre eles é realizada.

\section{Introdução}

Devido ao rápido desenvolvimento da IoT (Internet of Things) enquanto uma estratégia de ubiquidade, e sua utilização em diferentes aspectos da vida cotidiana, observa-se um aumento na quantidade de dados de contexto que continuamente são gerados, os quais representam o estado do ambiente ao longo do tempo. Acredita-se que a utilização efetiva deste grande volume de dados de contexto pode introduzir novas possibilidades para $o$ desenvolvimento humano, com base em soluções computacionais que sejam autônomas, responsivas e adaptativas ao contexto [Li et al. 2015]. 
Desta forma, a ciência de contexto torna-se uma abordagem chave para fornecer serviços adaptáveis, por exemplo, quando os serviços mais adequados são selecionados de acordo com as informações de contexto relevantes para o usuário, ou quando é necessário alterar os parâmetros operacionais dos serviços, em função do contexto, durante sua execução. Deste modo, as aplicações cientes de contexto devem ser capazes de adaptar seus comportamentos em mudança com um mínimo de intervenção humana, e para tanto introduzem desafios diversos para os seus desenvolvedores [Khattak et al. 2014].

Os sistemas cientes de contexto na sua operação envolvem uma grande quantidade de informações de contexto que precisam ser: recuperadas constantemente; efetivamente interpretadas; rapidamente processadas; divulgadas às aplicações interessadas; e mantidas em repositórios de contexto [Bellavista et al. 2012]. Portanto, para minimizar os esforços de desenvolvimento deste tipo de aplicação, precisam ser adotados mecanismos robustos para gerenciamento de contexto, bem como, esquemas de representação de contexto que possibilitem o tratamento da heterogeneidade dos dados envolvidos.

Investigar o gerenciamento de contexto, em termos de abordagens para modelagem de informações de contexto e estratégias de raciocínio para processamento das informações de contextuais, constitui parte de um significativo esforço de pesquisa. Além disso, é necessário investigar o emprego da ciência de contexto para o desenvolvimento de aplicações que são adaptáveis segundo os interesses definidos pelos usuários [Bibri 2015].

Uma série de abordagens de modelagem e estratégias para raciocínio de contexto foram recentemente desenvolvidas, no entanto, as mesmas não se mostram versáteis para atender, individualmente, os requisitos de raciocínio para as diferentes aplicações ubíquas, no cenário da IoT. Assim, identifica-se a necessidade de utilização de diferentes métodos para raciocínio de contexto, constituindo assim, as estratégias híbridas [Li et al. 2015].

O uso de estratégias híbridas para raciocínio é uma área de pesquisa em crescimento na comunidade devido a sua possibilidade de maior precisão preditiva. Ele fornece as abordagens adequadas para a solução baseada em computador considerando problemas complexos, como os que são enfrentados pelas aplicações ubíquas [Subbaraj and Venkataraman 2016].

O objetivo deste artigo é construir o estado da arte na área de ciência de contexto, empregando estratégias híbridas para raciocínio. Para tanto, é realizada uma Revisão Sistemática da Literatura (RSL), cujos procedimentos necessários para sua consecução são discutidos, facultando sua reconstrução com outros critérios. Como produto, foram identificados trabalhos representativos na área no período entre 2012 e 2017, sendo registrados os retornos obtidos nas diferentes bases de indexação. Ao final da RSL, foram selecionados sete trabalhos que propõem estratégias híbridas para raciocínio de contexto. Os trabalhos selecionados são apresentados e discutidos, sendo, ainda, realizada uma comparação entre eles.

Este artigo está dividido da seguinte forma: a Seção 2 discute a RSL realizada; a Seção 3 apresenta os trabalhos selecionados durante revisão, onde são analisadas as principais características de cada trabalho; e, por fim, a Seção 4 discute as considerações finais e os trabalhos futuros. 


\section{Trabalhos que propõem estratégias híbridas para raciocínio de contexto}

A RSL é uma metodologia de pesquisa que prevê a execução de uma série de procedimentos para gerar uma revisão de literatura em uma área, ou seja, identificar um conjunto de artigos que traz uma prospecção criteriosa do tema de pesquisa abordado [Kitchenham and Charters 2007]. Uma característica desta metodologia de pesquisa é que todos os seus procedimentos são registrados, permitindo que o estudo realizado seja reproduzível por outros pesquisadores.

\subsection{Questões de pesquisa}

A primeira etapa para o desenvolvimento de uma RSL é a definição das questões de pesquisa que fundamentam a mesma [Petersen et al. 2015]. Desta forma, as questões de pesquisa que fundamentam essa RSL e nortearam o estudo são:

RQ1: Quais estratégias para raciocínio de contexto são utilizadas em conjunto?

RQ2: Em quais domínios são aplicadas as estratégias híbridas para raciocínio?

RQ3: Que metodologia é empregada na avaliação?

\subsection{Processo de busca}

O processo de busca pelos artigos seguiu o fluxo de execução apresentado na figura 1, onde pode ser visualizado que o processo possuiu quatro etapas. A primeira etapa é a aplicação da string de busca na bases de indexação selecionadas. Na segunda etapa é realizado o primeiro filtro, o qual elimina os trabalhos publicados antes de 2012, analisando assim somente trabalhos dos últimos cinco anos, desta forma, buscando os trabalhos publicados entre 2012 e 2017 . A terceira etapa é responsável por realizar um filtro com base na string de busca, analisando o título, resumo e palavra-chave dos trabalhos. A ultima etapa é responsável por analisar todo o texto, tendo como base os critérios de inclusão e exclusão, sendo selecionados somente os artigos que propõem estratégias híbridas para raciocínio de contexto.

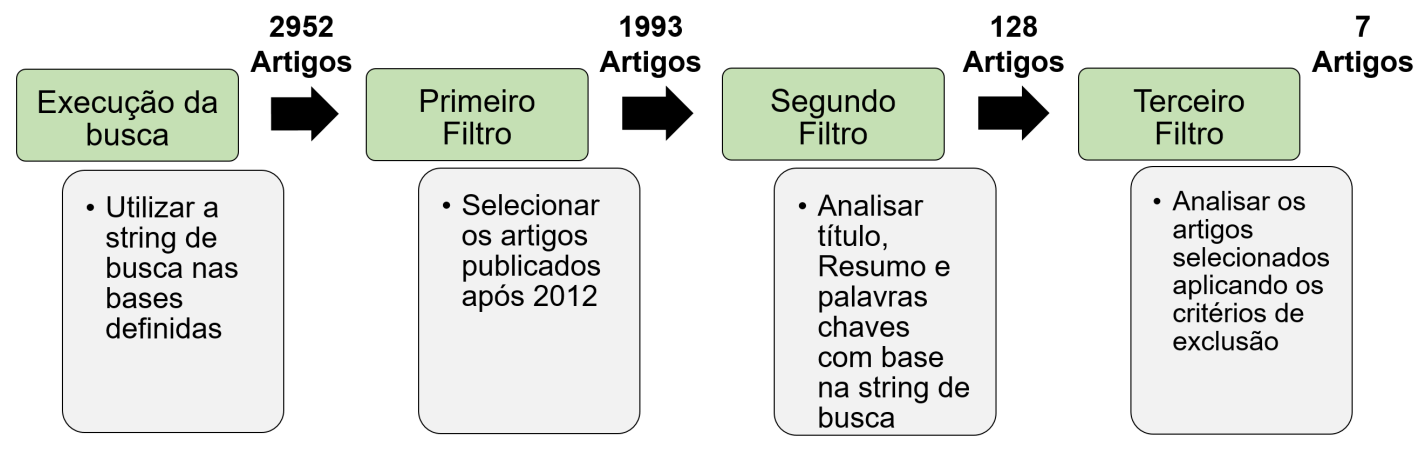

Figura 1. Fluxo do processo de revisão sistemática.

A string de busca utilizada no processo de revisão sistemática da literatura foi: ((“hybrid reasoning") OR ("hybrid approach") OR ("hybrid framework") OR ("hybrid strategy")) AND ( (“context aware") OR (“context awareness")).

A fim de encontrar os estudos relevantes foi aplicada a string de busca em seis bases de indexação: (i) ACM; (ii) IEEE; (iii) Science Direct; (iv) Scopus; (v) Springer; (vi) Web of Science. A escolha das bases de indexação se deu devido a sua importância, bem como, por cobrirem a maioria dos periódicos e artigos de conferências publicados no campo da ciência da computação, com foco em aplicações cientes de contexto. 


\subsection{Critérios de exclusão}

Os filtros aplicados nesta RSL seguem os seguintes critérios de exclusão:

- trabalhos publicados antes de 2012;

- trabalhos que não contêm a string de busca no título, resumo ou palavras-chave;

- trabalhos que não são artigos publicados em conferências ou periódicos;

- trabalhos que não estão escritos em inglês ou português;

- trabalhos que não apresentam estratégias para raciocínio;

- trabalhos que propõem estratégias para raciocínio que não são híbridas;

- trabalhos que registram pequenas diferenças de outro artigo.

O último critério de exclusão foi utilizado para excluir os trabalhos preliminares de uma mesma pesquisa, sendo selecionado somente o trabalho mais atual. Para aplicação desse critério foi analisado todo o texto dos artigos, verificando se os artigos tratavam da mesma pesquisa, registrando apenas pequenas diferenças entre eles.

\subsection{Resultados da revisão sistemática}

A figura 2 apresenta uma visão geral dos artigos retornados por cada base de indexação durante o processo de revisão sistemática empregado. Destaca-se que os artigos duplicados estão contabilizados na figura.

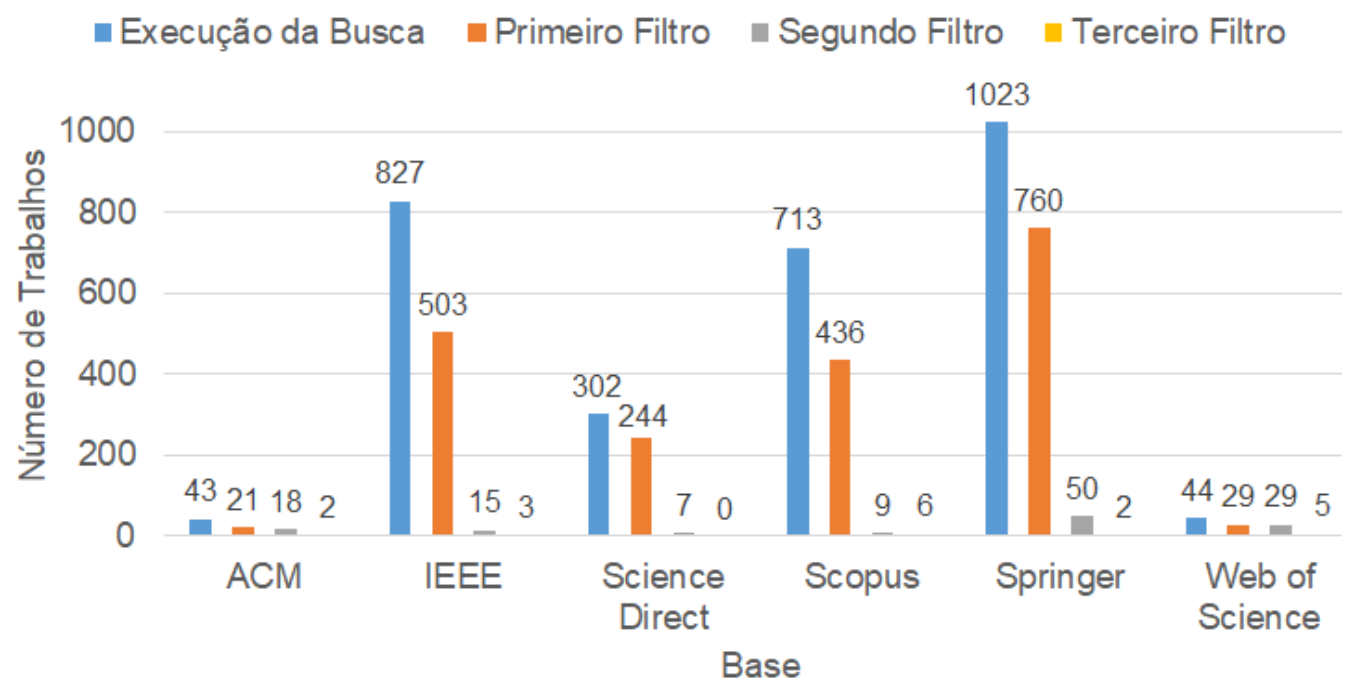

Figura 2. Artigos retornados em cada base de indexação.

A tabela 1 apresenta uma visão geral do número de artigos que foram excluídos com base em cada critério de exclusão. Conforme pode ser visualizado, o critério que excluiu o maior número de trabalhos foi de analisar o título, resumo e palavra-chave tendo como base a string de busca, sendo excluídos 1865 artigos.

\section{Trabalhos selecionados na RSL realizada}

Após a realização da RSL apresentada anteriormente, foram selecionados 7 artigos, os quais são apresentados a seguir, sendo explorados aspectos referentes a sua estratégia para raciocínio, bem como, suas principais funcionalidades. 
Tabela 1. Número de artigos excluídos por cada critério de exclusão.

\begin{tabular}{c|c} 
Critério de Exclusão & Número de Artigos Excluídos \\
\hline Trabalhos publicados antes de 2012 & 959 \\
\hline Trabalhos que não contêm a string de busca no título, resumo ou palavras-chave & 1865 \\
\hline Trabalhos que não são artigos publicados em conferências ou periódicos & 10 \\
\hline Trabalhos que não são escritos em inglês ou português & 2 \\
\hline Trabalhos que não apresentam estratégias para raciocínio de contexto & 24 \\
\hline Trabalhos que propõem estratégias para raciocínio que não são híbridas & 11 \\
\hline Trabalhos que apresentam pequenas modificações de outro artigo & 4 \\
\hline
\end{tabular}

\subsection{Raciocínio baseado em regras e em ontologias}

O artigo de [Lopes et al. 2012] propõe uma arquitetura para ciência do contexto, a qual tem como premissa fundamental a possibilidade de interpretação e a composição dinâmica das informações adquiridas por sensores posicionados em diferentes localidades do ambiente ubíquo. Com isso, a arquitetura busca viabilizar a construção de contextos complexos, cujas informações contextuais possam ser obtidas de forma distribuída, bem como ter suas regras de processamento alteradas sem a necessidade de interromper a execução das aplicações.

Para realização do processamento das informações contextuais é proposta uma estratégia híbrida para raciocínio a qual utiliza um raciocínio semântico provido pela ontologia, com base em axiomas e regras para inferir novos conhecimentos baseadas nas instâncias da ontologia. Além deste, é proposta a utilização de um raciocínio baseado em regras do tipo ECA (Evento-Condição-Ação), o qual realiza o raciocínio de informações contextuais providas por um modelo relacional.

\subsection{Raciocínio baseado em ontologias e em casos}

Em [Strobbe et al. 2012] é proposta uma plataforma que tem como principais características ser ciente de contexto e tratar da agregação e da abstração de informações de contexto usando ontologias para representar as mesmas. A plataforma CASP utiliza na etapa de processamento de contexto dois raciocinadores, um baseado em regras ontológicas e o outro em casos.

Para viabilizar o raciocínio híbrido, são exploradas combinações dos raciocinadores baseados em regras e em casos. As informações de contexto de alto nível podem ser derivadas usando regras, casos ou uma combinação de ambos. As regras ontológicas representam conhecimento geral do domínio, enquanto os casos capturam conhecimento específico. O raciocínio baseado em regras avalia as regras fornecidas e adiciona as informações derivadas à base de conhecimento. $\mathrm{O}$ raciocínio baseado em casos recupera as situações semelhantes e usa as soluções correspondentes para atualizar as informações na base de conhecimento representado pelo modelo ontológico.

\subsection{Raciocínio baseado em regras fuzzy e em casos}

O artigo [Yuan and Herbert 2014] apresenta um framework para raciocínio híbrido desenvolvido para o sistema CARA (Context-Aware Real-time Assistant). O framework realiza 
o monitoramento de um ambiente doméstico inteligente, fornecendo uma fusão de dados cientes de contexto, bem como mecanismos de detecção de anomalias que suportam a atividade de análise e geração de alertas.

O raciocínio híbrido é baseado em regras fuzzy e em casos, permitindo tornar o CARA mais robusto e adaptável a um ambiente sujeito a mudança. Emprega-se o uso do raciocínio baseado em casos para detectar anomalias condicionais para automação residencial e o uso do raciocínio baseado em regras difusas hierárquicas para lidar com exceções, bem como, para recuperação de casos e adaptação de casos sensíveis à consulta.

\subsection{Raciocínio baseado em aprendizagem de máquina}

Em [Smaaberg et al. 2014] é apresentado um protótipo de um sistema de recomendação de concertos para grupos de usuários. O protótipo é ciente de contexto e leva em conta a localização e a temporalidade do usuário ao dar recomendações. Para realizar o raciocínio das informações contextuais é proposta uma estratégia híbrida a qual implementa dois algoritmos, os quais aproveitam as informações históricas dos usuários, um algoritmo de linhagem colaborativa (K-Nearest Neighbor) e um algoritmo de fatoração de matriz. Os algoritmos podem ser utilizados de forma individual ou combinada.

A usabilidade do protótipo foi avaliada usando a Escala de Usabilidade do Sistema e uma avaliação centrada no usuário foi realizada para avaliar a qualidade das recomendações. O objetivo do protótipo era recomendar concertos para um usuário dentro da localização e de um tempo específico, com o intuito de oferecer recomendações que os usuários poderiam ter interesse em participar. Os resultados da avaliação de usabilidade mostram que os usuários ficaram satisfeitos com a usabilidade do protótipo. Os resultados da Avaliação da Qualidade mostram que a abordagem que combina os dois algoritmos obteve o melhor resultado. O protótipo foi testado e os usuários ficaram satisfeitos com a qualidade das recomendações.

\subsection{Raciocínio baseado em aprendizagem de máquina e lógica probabilística}

No artigo [Baz et al. 2016] é proposto um sistema que classifica os produtos de varejo em uma prateleira de loja. O sistema de classificação proposto combina os pontos fortes de classificadores sem contexto e informações de contexto. O objetivo do sistema é projetar um modelo probabilístico que codifique as relações entre os produtos na prateleira e combine isso com os métodos atuais de classificação de imagem baseada em visão.

São propostos duas estratégias híbridas diferentes, a primeira estratégia híbrida combina SVMs (Support Vector Machines) e um modelo gráfico generativo que tenta explicitamente modelar uma distribuição de probabilidade conjunta, com base em HMMs (Hidden Markov Models). Na segunda estratégia, os SVMs são combinados com uma abordagem discriminativa baseada em CRFs (Conditional Random Fields) tendo como intuito formar um novo contexto. Os modelos probabilísticos são treinados aprendendo com os erros do classificador livre de contexto (SVMs) e as relações vizinhas entre os produtos de varejo.

\subsection{Raciocínio baseado em ontologia e aprendizagem de máquina}

Em [Razzaq et al. 2017] é proposta uma estratégia híbrida para raciocínio de contexto para um framework que oferece suporte personalizado para saúde e bem-estar. Para realizar o raciocínio das informações contextuais é proposta uma nova abordagem conjuntiva, 
a qual é baseada em ontologia e aprendizagem de máquina. A estratégia baseada em aprendizagem de máquina suporta a inferência classificando contextos de alto nível com base no conjunto de dados instanciado na ontologia.

Para realização do armazenamento dos dados de contexto providos pela ontologia os autores propõem a utilização do modelo de triplas. Nos testes realizados foi combinada uma estratégia baseada em ontologia com seis diferentes técnicas de aprendizagem de máquina: Naive Bayes, KStar, IBK, J48, RandomForest e RandomTree. A técnica que combinada com a ontologia apresentou o melhor resultado foi a RandomForest, enquanto que a KStar apresentou o pior resultado.

\subsection{Raciocínio baseado em ontologias e lógica probabilística}

No artigo [Li et al. 2017] é proposta uma estratégia híbrida para o raciocínio de contexto para um framework de ciência de contexto. A estratégia proposta é constituída de três técnicas de raciocínio: (i) ontológico, sendo utilizados axiomas internos à ontologia com o intuito de inferir conhecimento relacionado as classes presentes na mesma; (ii) baseado em regras, que consiste de regras que processam os dados contextuais presentes na ontologia, sendo utilizada a linguagem SWRL (Semantic Web Rule Language) para a concepção das regras; (iii) Multi-Entity Bayesian Network, o qual consiste de um sistema lógico que integra a lógica da primeira ordem com a teoria da probabilidade bayesiana.

Os autores destacam que, com a incorporação das três técnicas, é proporcionado um melhor desempenho para o raciocínio de contexto, aproveitando-se dos pontos positivos de cada técnica, com o intuito de compensar as fraquezas das mesmas. Os autores destacam que a estratégia híbrida proposta é eficaz para fornecer diferentes capacidades de raciocínio e satisfazer as diferentes necessidades das aplicações [Li et al. 2017].

\subsection{Discussão dos trabalhos selecionados na RSL}

A tabela 2 apresenta uma análise comparativa entre os trabalhos selecionados na RSL realizada. Esta análise é realizada com base nos seguintes critérios: (i) os tipos de sensores empregados na etapa de aquisição; (ii) os modelos utilizados para realizar a representação do contexto; (iii) as técnicas presentes para realizar o processamento; (iv) a forma de armazenamento dos dados contextuais; e (v) algumas ferramentas utilizadas para o desenvolvimento do trabalho.

Com base na tabela 2 é constatado que na etapa de aquisição apenas dois trabalhos não deixam claro como fazem a aquisição dos dados de contexto. Enquanto os outros trabalhos analisados possuem suporte para lidar com diferentes tipos de sensores, assim permitindo os mesmos atender à aplicações de diferentes naturezas.

Analisando a etapa de modelagem, pode ser visualizado que quatro trabalhos utilizam o modelo baseado em ontologias, principalmente devido a sua característica de representação semântica. Destaca-se ainda que em [Lopes et al. 2012] foi proposta a utilização de uma modelagem híbrida combinando modelos ontológicos e relacionais.

Em relação ao armazenamento das informações contextuais, nota-se que a maioria dos trabalhos não discute informações referentes a esta funcionalidade, apesar da mesma ser importante para as aplicações cientes de contexto. O trabalho de [Yuan and Herbert 2014] utiliza o modelo baseado em linguagem de marcação, empregando a linguagem XML (eXtensible Markup Language), principalmente pela facilidade 
Tabela 2. Comparação entre os trabalhos selecionados.

\begin{tabular}{|c|c|c|c|c|c|}
\hline Trabalhos & Aquisição & Modelo & Processamento & Armazenamento & Ferramentas \\
\hline LOPES et al., 2012 & $\begin{array}{l}\text { Sensores } \\
\text { Físicos } \\
\text { Lógicos }\end{array}$ & $\begin{array}{l}\text { Ontológico e } \\
\text { Relacional }\end{array}$ & $\begin{array}{c}\text { Regras e } \\
\text { Regras Ontológicas }\end{array}$ & Relacional & $\begin{array}{c}\text { Java, Jena, OSGI, } \\
\text { Protégé, Pellet, } \\
\text { PostgreSQL }\end{array}$ \\
\hline $\begin{array}{c}\text { STROBBE et al., } \\
2012\end{array}$ & $\begin{array}{l}\text { Sensores } \\
\text { Físicos } \\
\text { Lógicos }\end{array}$ & Ontológico & $\begin{array}{l}\text { Regras Ontológicas e } \\
\text { Baseado em Casos }\end{array}$ & $\begin{array}{l}\text { Informação } \\
\text { Não } \\
\text { Disponível }\end{array}$ & $\begin{array}{c}\text { Java, Jena, OWL API, } \\
\text { jCOLIBRI }\end{array}$ \\
\hline $\begin{array}{l}\text { YUAN; HERBERT, } \\
2014\end{array}$ & $\begin{array}{l}\text { Sensores } \\
\text { Físicos } \\
\text { Lógicos }\end{array}$ & Fuzzy & $\begin{array}{c}\text { Regras Fuzzy e } \\
\text { Baseado em Casos }\end{array}$ & XML & jCOLIBRI \\
\hline $\begin{array}{c}\text { SMAABERG; } \\
\text { SHABIB; KROGSTIE, } \\
\text { J., } 2014\end{array}$ & $\begin{array}{l}\text { Informação } \\
\text { Não } \\
\text { Disponível }\end{array}$ & $\begin{array}{l}\text { Informação } \\
\text { Não } \\
\text { Disponível }\end{array}$ & $\begin{array}{c}\text { Aprendizagem de } \\
\text { Máquina (KNN e } \\
\text { Fatoração de Matriz) }\end{array}$ & $\begin{array}{l}\text { Informação } \\
\text { Não } \\
\text { Disponível }\end{array}$ & $\begin{array}{c}\text { JavaScript, HTML5, } \\
\text { Durandal.js, Model } \\
\text { View }\end{array}$ \\
\hline $\begin{array}{l}\text { BAZ; YORUK; } \\
\text { CETIN, } 2016\end{array}$ & $\begin{array}{l}\text { Informação } \\
\text { Não } \\
\text { Disponível }\end{array}$ & $\begin{array}{l}\text { Informação } \\
\text { Não } \\
\text { Disponível }\end{array}$ & $\begin{array}{l}\text { Aprendizagem de } \\
\text { Máquina e Lógica } \\
\text { Probabilística }\end{array}$ & $\begin{array}{l}\text { Informação } \\
\text { Não } \\
\text { Disponível }\end{array}$ & VLFEAT toolbox \\
\hline $\begin{array}{l}\text { RAZZAQ; AMIN; } \\
\text { LEE, } 2017\end{array}$ & $\begin{array}{l}\text { Sensores } \\
\text { Físicos } \\
\text { Lógicos }\end{array}$ & Ontológico & $\begin{array}{l}\text { Aprendizagem de } \\
\text { Máquina e Axiomas } \\
\text { Ontológicos }\end{array}$ & $\begin{array}{c}\text { Jena TDB (triple } \\
\text { store) }\end{array}$ & $\begin{array}{c}\text { Java, Maven, Jena, } \\
\text { Protégé, Pellet, } \\
\text { OWL-API Weka }\end{array}$ \\
\hline $\begin{array}{l}\text { LI; MARTÃNEZ; } \\
\text { RUBIO, } 2017\end{array}$ & $\begin{array}{l}\text { Sensores } \\
\text { Físicos } \\
\text { Lógicos }\end{array}$ & Ontológico & $\begin{array}{l}\text { Regras Ontológicas, } \\
\text { Axiomas Ontológicos e } \\
\text { Lógica Integrada com } \\
\text { Probabilidade } \\
\text { Bayesiana }\end{array}$ & $\begin{array}{l}\text { Informação } \\
\text { Não } \\
\text { Disponível }\end{array}$ & Pellet \\
\hline
\end{tabular}

da mesma ser utilizada para transmitir as informações entre diferentes componentes. Enquanto o trabalho de [Lopes et al. 2012] emprega um modelo relacional de armazenamento, o qual não é considerado um modelo satisfatório para o armazenamento de dados providos pelo modelo ontológico [Can et al. 2017]. Além destes, o trabalho de [Razzaque et al. 2016] propõe a utilização do modelo de triplas, sendo um modelo mais propício para realizar a persistência das informações providas por ontologias.

Acredita-se serem necessários mecanismos com diferentes características para armazenamento de contexto, já que as aplicações cientes de contexto atuais tendem a lidar com diferentes tipos de dados, níveis semânticos entre outros aspectos. Por sua vez, a eficiência destes mecanismos é um aspecto significativo, considerando a escalabilidade do volume de dados contextuais envolvido, sua constante atualização ou consulta.

No que diz respeito ao raciocínio de contexto, os trabalhos apresentados apesar de utilizarem estratégias híbridas, os mesmos não permitem as diferentes aplicações comporem as estratégias que serão utilizadas em função dos dados contextuais envolvidos. Desta forma, nota-se a ausência de uma abordagem que permita a combinação das diferentes estratégias para o raciocínio de contexto. Onde a criação desta abordagem poderá aumentar a flexibilidade para as aplicações utilizarem os contextos de interesse, como também facilitar a identificação das situações de interesse. 
Com base na análise dos artigos selecionados na RSL realizada é possível responder as questões de pesquisa que fundamentaram esta revisão. As questões de pesquisa e as repostas adquiridas são:

RQ1: Quais estratégias para raciocínio de contexto são utilizadas em conjunto? Foi possível observar que não existe um padrão para efetivação da combinações das estratégias, por exemplo, estratégias baseadas em ontologias são usados com regras, casos e aprendizagem de máquina. Ressalta-se que o trabalho de [Smaaberg et al. 2014] combina três técnicas diferentes, entretanto todas baseada em aprendizagem de máquina.

RQ2: Em quais domínios são aplicadas as estratégias híbridas para raciocínio? Dentre os sete trabalhos selecionados, três deles [Lopes et al. 2012, Strobbe et al. 2012, Yuan and Herbert 2014] se caracterizam por poderem ser aplicados em diferentes domínios. Enquanto os outros trabalhos [Smaaberg et al. 2014, Baz et al. 2016, Razzaq et al. 2017, Li et al. 2017] são aplicados respectivamente em: sistemas de recomendação; sistemas de classificação; paradigma de saúde e bem estar; robôs subaquáticos.

RQ3: Que metodologia é empregada na avaliação? Os trabalhos utilizaram uma metodologia baseada em cenários de uso para a sua avaliação. Dentre os trabalhos que propuseram a utilização técnicas de aprendizagem de máquina, dois deles [Smaaberg et al. 2014, Baz et al. 2016] utilizaram uma base de dados para realização da avaliação, enquanto [Razzaq et al. 2017] realizou a coleta de dados de 20 usuários, gerando sua própria base de dados.

\section{Considerações finais}

Este artigo construiu o estado da arte, sistematizando tendências de pesquisa em Ciência de Contexto empregando estratégias híbridas para raciocínio. Para tanto, é explorada uma Revisão Sistemática da Literatura, contribuindo tanto pela identificação de trabalhos relevantes na área, bem como, pela especificação de critérios para sua seleção. Nesta revisão foram analisados trabalhos publicados nos últimos cinco anos, analisando os trabalhos entre 2012 e 2017. Durante o processo de revisão foram identificados 2952 trabalhos, sendo selecionados ao final sete trabalhos que propõem estratégias híbridas. Os trabalhos selecionados são apresentados, discutidos, e uma comparação entre eles é realizada.

Na comparação realizada foram discutidos os recursos oferecidos pelos trabalhos para tratar a aquisição, modelagem, processamento e armazenamento de informações contextuais, bem como, as ferramentas utilizadas em sua prototipação.

Como trabalho futuro destaca-se a concepção de um framework flexível para provimento da ciência de contexto para as aplicações ubíquas. Este framework a ser concebido deverá ser flexível no que diz respeito aos recursos oferecidos, disponibilizando uma abordagem para raciocínio de contexto que permita uma composição dinâmica de diferentes estratégias.

\section{Agradecimentos}

O presente trabalho foi realizado com apoio da CAPES (Programa Nacional de Cooperação Acadêmica - Procad) e da FAPERGS (Programa Pesquisador Gaúcho - PqG). Roger S. Machado é Bolsista FAPERGS/CAPES - BRASIL, nível doutorado. 


\section{Referências}

Baz, I., Yoruk, E., and Cetin, M. (2016). Context-aware hybrid classification system for fine-grained retail product recognition. In IEEE 12th IVMSP, France.

Bellavista, P., Corradi, A., Fanelli, M., and Foschini, L. (2012). A survey of context data distribution for mobile ubiquitous systems. ACM Comput. Surv., 44(4):24:1-24:45.

Bibri, S. E. (2015). Context modeling, representation, and reasoning: An ontological and hybrid approach. In The Human Face of Ambient Intelligence, volume 9 of Atlantis Ambient and Pervasive Intelligence, pages 197-257. Atlantis Press, Paris.

Can, O., Sezer, E., Bursa, O., and Unalir, M. O. (2017). Comparing relational and ontological triple stores in healthcare domain. Entropy, 19(1):30.

Khattak, A. M., Akbar, N., Aazam, M., Ali, T., Khan, A. M., Jeon, S., Hwang, M., and Lee, S. (2014). Context representation and fusion: Advancements and opportunities. Sensors, 14(6):9628-9668.

Kitchenham, B. and Charters, S. (2007). Guidelines for performing systematic literature reviews in software engineering. Joint report, Keele University and Durham University.

Li, X., Eckert, M., Martinez, J.-F., and Rubio, G. (2015). Context aware middleware architectures: Survey and challenges. Sensors, 15(8):20570.

Li, X., Martínez, J.-F., and Rubio, G. (2017). Towards a hybrid approach to context reasoning for underwater robots. Applied Sciences, 7(2).

Lopes, J., Souza, R., Gadotti, G., Gusmão, M., Costa, C., Barbosa, J., Yamin, A., and Geyer, C. (2012). A distributed architecture for dynamic contexts composition in ubicomp. In 38th CLEI 2012 - Conference Proceedings, Colombia.

Petersen, K., Vakkalanka, S., and Kuzniarz, L. (2015). Guidelines for conducting systematic mapping studies in software engineering: An update. Information and Software Technology, 64(Supplement C):1 - 18.

Razzaq, M. A., Amin, M. B., and Lee, S. (2017). An ontology-based hybrid approach for accurate context reasoning. In 19th APNOMS, pages 403-406, South Korea.

Razzaque, M., Milojevic-Jevric, M., Palade, A., and Clarke, S. (2016). Middleware for internet of things: A survey. Internet of Things Journal, IEEE, 3(1):70-95.

Smaaberg, S., Shabib, N., and Krogstie, J. (2014). A user-study on context-aware group recommendation for concerts. In CEUR Workshop Proceedings - SP 2014, Chile.

Strobbe, M., Van Laere, O., Dhoedt, B., De Turck, F., and Demeester, P. (2012). Hybrid reasoning technique for improving context-aware applications. Knowledge and Information Systems, 31(3):581-616.

Subbaraj, R. and Venkataraman, N. (2016). Reasoning in context aware computing - a review. International Journal of Pharmacy and Technology, 8:5021-5032.

Yuan, B. and Herbert, J. (2014). Context-aware hybrid reasoning framework for pervasive healthcare. Personal and Ubiquitous Computing, 18(4):865-881. 